\title{
Oral manifestations of type 2 diabetes mellitus of Bhopal city: an observational prospective study
}

\author{
Saxena $V^{1}$, Saxena $D^{2}$, Desai $A^{3}$, Shrivastava $S^{4}$ \\ ${ }^{1}$ Dr. Vijay Saxena, Associate Professor, Department of Medicine, People's College of Medical Sciences \& Research \\ Center, Bhanpur, Bhopal, ${ }^{2}$ Dr. Divya Saxena, Senior lecturer, Department of Conservative Dentistry and Endodontics, \\ Mansarovar Dental College, Hospital and Research Centre, Bhopal, M.P, ${ }^{3}$ Dr. Ami Desai, Senior Lecturer, Department \\ of Oral Pathology and Microbiology, People's College of Dental Sciences \& Research Center, Bhanpur, Bhopal, M.P., \\ ${ }^{4}$ Dr. Saurabh Shrivastava, M.D.S. Senior Lecturer, Department of Prosthodontics and Crown \& Bridge and \\ Implantology, Mansarovar Dental College, Hospital and Research Centre, Bhopal, M.P, India.
}

Address for Correspondence: Dr. Ami Desai, 26- Sai Hills, Kolar Road, Bhopal, M.P. India. e-maildramisaxena@gmail.com

\begin{abstract}
Introduction: Type 2 diabetes mellitus involves a variety of dysfunctions bringing about a few delicate tissue variations from the norm in the oral cavity. These incorporate gingivitis periodontitis, salivary dysfunction, taste dysfunction, oral fungal and bacterial infections, geographic tongue, benign migratory glossitis, fissured tongue, traumatic ulcer, lichen planus, angular cheilitis, delayed mucosal healing, dental root caries, tooth loss, mucosal neurosensory disorders, and oral mucosal lesions. Aim and objectives: This study aims to determine the frequency of different oral manifestations in patients of Type 2 Diabetes Mellitus of Bhopal city which will expand the consciousness of oral signs of diabetes mellitus subsequently helping in speeding up the examination regarding this matter. Methodology: This descriptive study was carried out at the Department of Medicine, People's Hospital, hitech division, Bhopal from March 2016 to January 2017. 200 patients satisfying the inclusion criteria (diagnosed cases of type 2 diabetes mellitus of > 35 years of age) were included in the study. $84(42 \%)$ were female and $116(58 \%)$ were male patients. Result: The frequency of different oral manifestations seen in female diabetic patients in descending order were; gingivitis (49\%), periodontitis (36.7\%), dental root caries $(20 \%)$, taste dysfunction (8\%), burning mouth syndrome (8\%), xerostomia (31\%), recurrent apthous stomatitis $(11 \%)$ and oral candidiasis $(2 \%)$ and in male diabetic patients in descending order were; gingivitis $(41 \%)$, periodontitis $(29 \%)$, dental root caries $(18 \%)$, taste dysfunction (9\%), burning mouth syndrome (3\%), xerostomia $(31 \%)$, recurrent apthous stomatitis $(5 \%)$ and oral candidiasis $(3 \%)$. Conclusion: It was inferred that diabetic patients are more inclined to get oral complications so health education, timely diagnosis and effective treatment should be carried out to prevent these complications.
\end{abstract}

Key words: Diabetes mellitus, Oral manifestations and complications

\section{Introduction}

Diabetes Mellitus comprises of common metabolic disorders that share the phenotype of hyperglycemia. ${ }^{1}$ The two broad categories are type 1 and type 2 . Type 2 diabetes mellitus is characterized by variable degree of insulin resistance; impaired insulin secretion thus increased glucose production. Based on the current trends, >360 million individuals will have diabetes by

Manuscript received: $4^{\text {th }}$ March 2017

Reviewed: $11^{\text {th }}$ March 2017

Author Corrected: $18^{\text {th }}$ March 2017

Accepted for Publication: $25^{\text {th }}$ March 2017 the year 2030 [1,2]. The prevalence of type 2 diabetes mellitus is rising much more rapidly because of change in the life style pattern in developed and industrialized countries [2-5]. Various oral disorders have been linked with diabetes mellitus [3-6]. The association of diabetes mellitus and periodontal diseases (such as gingivitis and periodontitis) has received the greatest attention.

In addition to gingivitis and periodontitis, root dental caries, salivary dysfunction, oral mucosal diseases, oral 
infections such as candidiasis, taste and other neurosensory disorders are also found in patients of diabetes mellitus [3]. In Patients of type 2 diabetes mellitus which often is associated with obesity and intake of high-calorie and carbohydrate-rich foodcan be expected to have a greater exposure to cariogenic foods [3,7-9].

Furthermore, reduced salivary flow has been reported in people with diabetes who have neuropathy [4] and diminished salivary flow is a risk factor for causation of dental caries.

Diabetes is believed to advance periodontitis through an exaggerated inflammatory reaction to the periodontal microflora [6-9].

Dry mouth, or xerostomia, has been found in people with diabetes mellitus [4]. Salivary dysfunction, however, can be difficult to diagnose.

A number of typesof oral mucosal lesions, including lichen planus and recurrent aphthous stomatitis, have been reported in individuals having diabetes mellitus.

Not all study results have presented this association, and these are moderately common disorders that often are seen in patients who do not have diabetes [7-9].

Conversely, oral candidiasis has been a more common presentation in patients with diabetes [6].

Candidiasis is an outcome of an immunocompromised state, and a diminishment in salivary flow is another risk factor for oral candidiasis [10].

Taste disturbances is a complex symptom which may be related to salivary flow and changes in food intake associated with disease management.

Other neurosensory disorders of the oral and perioral tissues, include burning mouth syndrome and dysphagia, have been observed in patients with diabetes [3, 6-9]. Unlike other reported oral manifestations of diabetes mellitus, periodontal ailment is a recognized and well documented complication of diabetes mellitus. Data suggest that periodontal disease may enhance the risk of experiencing poor metabolic control [2, 3, 7].

This study primarily aims to determine the incidence of diverse oral manifestations in Type 2 Diabetes Mellitus patients of Bhopal city which will subsequently expand the consciousness of oral signs of diabetes mellitus as well as aids in early detection and examination regarding this matter.

\section{Methodology}

Study design and setting- This study was conducted at the Department of Medicine, People's Hospital, hitech division, Bhopal from March 2016 to January 2017. It was a cross sectional prospective/observational study.

Information such as patient age, gender, address and any other relevant medical information were recorded on a predesigned Performa by the author, followed by detailed dental history, intra-oral examination and radiographs. All the data were collected through the specially designed Performa.

Participants- 200 patients diagnosed as suffering from type 2 diabetes mellitus of $>35$ years of age were included in the study.

Inclusion and exclusion criteria- Patients agreeable to provide written informed consent were included in the study. Physically and mentally incapacitated patients, patients of Type 1 diabetes mellitus, patients of chronic renal failure additionally patients on immunosuppressive therapy or who had undergone any transplantation and those using alcohol/smoking were excluded.

Statistical method- Data were analyzed in terms of frequency, percentages using SPSS 15. Mean and standarddeviation was calculated for age, while only percentage was calculated for gender.

\section{Results}

Out of two hundred patients $84(42 \%)$ were female and $116(58 \%)$ were male patients. The mean age of the patients was 54.36 years ( $\mathrm{SD} \pm 3.84)$. Minimum age was 39 years and maximum age was 78 .

The frequency of different oral manifestations and complications seen in these patients are shown in table 1: 
Table-1: Oral manifestations of diabetes mellitus.

\begin{tabular}{|c|c|c|c|c|}
\hline Oral manifestation & \multicolumn{2}{|c|}{ Female (total number- 84) } & Male & (total number- 116) \\
\hline & numbers & Percentage & numbers & $41 \%$ \\
\hline Gingivitis & 41 & $49 \%$ & 48 & $29 \%$ \\
\hline Periodontitis & 31 & $37 \%$ & 34 & $18 \%$ \\
\hline Root caries & 17 & $20 \%$ & 6 & $5 \%$ \\
\hline Recurrent apthous stomatitis & 9 & $11 \%$ & 3 & $13 \%$ \\
\hline Candidiasis & 17 & $2 \%$ & 15 & $19 \%$ \\
\hline Xerostomia & 26 & $31 \%$ & 22 & $3 \%$ \\
\hline Taste dysfunction & 67 & $8 \%$ & 3 & $3 \%$ \\
\hline
\end{tabular}

\section{Discussion}

The oral signs and symptoms of the diabetes can be vital markers of the risk of both periodontal disease and further diabetic complications and symptoms associated with dental structures may furnish hints about the occurrence of diabetes [1, 3-6]. Periodontal disease was identified as the sixth complication of diabetes in 1993, and in the 1997 report of theExpert Committee on the Diagnosis and Classification of Diabetes Mellitus, periodontal disease was alluded to as one of the pathologic conditions frequently present in people with diabetes [1-4].

The relationship amongst diabetes and periodontal disease has been recognized in the dental literature for many decades. In fact many studies have confirmed that the prevalence, severity, and progression of periodontal disease are significantly increased in patients with diabetes [3-6]. Furthermore, longitudinal studies have demonstrated that severe periodontal disease indiabetic patients at baseline is related with poormetabolic control and other diabetes complications at follow-up.

Various reports on the relationship between diabetes and oral complications have included children and adolescents; however, these studies arelimited in terms of the depth of data collected andanalyses presented. Various authors have studied periodontal conditions in diabetics and observed that in patients with childhood onset diabetes, periodontitis seems to ensue around puberty and advances with age [4-6]. Previous studies have also shown that gingival inflammation is significantly increased as compere to non-diabetic control subjects, even after maintaining good oral hygiene levels. Findings from the present study demonstrate that periodontal destruction is increased in adolescents suffering from diabetes and, essentially is in direct proportion to the level of hyperglycemia, raised HbA1c duration of diabetes [5, 8, 12]. The etiopathogenesis of both diabetes and periodontitisis complex, and recognizing the mechanisms underlying this association was beyond the scope of the present study. However, evidence suggests that mechanisms that account for the development of other diabetes complications might be operating in the pathogenesis of accelerated periodontitis in diabetes as well [6-9]. According to previous evidence individuals having diabetes and poor metabolic controlare at a higher risk for suffering from more severe periodontitis.

However, not all studies have reported such an association, and conflicting data exist between prospective follow-up studies that have in their majority suggested the former and many cross sectional studies like this study that have shown no correlation $[6,8,12]$. Further studies including larger numbers of children and adolescents are required. These will reveal more insight into these associations, other factors involved, as well as the natural history of the development of periodontal changes in diabetes [10]. Dental caries, xerostomia, and mucosal lesions have been found in patients with diabetes, but thedata remains conflicting. Most recent studies suggest that young individuals with diabetes exhibit coinciding levels ofcaries to systemically healthy individuals [7, 9-11].

Present study has reported that diabetes is a major risk factor for the development of oral manifestations of diabetes mellitus, and it will not be appropriate to use the data available from, as confounding factors like alcohol use, literacy, diet, health education, obesity 
economical status etc can play important role in the aetiopathogenesis of these manifestations [9-12]. Therefore a lot of practice is to be done to decrease the frequency of these complications to reduce the morbidity in diabetic patients. Strong communication of dentist with diabetologist is a critical component while treating patients with diabetes.

Diabetologist must be aware of oral manifestations of the diabetes such as periodontitis and other and dentists must be updated on glycemic control in order to maintain a patient's oral health $[7,10]$. Treating patients with diabetes additionally represents anopportunity to grow a dental practitioner's referral base. Physicians who treat children and adults with diabetes could be a decent referral source of patients whose oral healthcare needs may not be fulfilled adequately [1, 3, 9-11]. Most forms of dental therapy ought not to interfere with the medical control of diabetes.

However, orofacial infections, dentoalveolar surgery and the stress level of dental procedures can increase serum glucose levels and metabolic insulin requirements [13, 14]. Therefore, dental practitioners must consider modifying and adjusting therapeutic treatment in consultation with the patient's diabetologist.

\section{Conclusion}

On the basis of the available information and data, it can be concluded that practicing dentists and dental hygienists may have a noteworthy positive effect on the oral and general wellbeing of patients with diabetes mellitus. Althoughmany aspects of this new segment of dental practice need to be developed, it is an opportunity, and the profession should embrace.

Prevention and management of oral manifestations, especially periodontal disease, in patients with diabetes is essential because to their possible adverse effect on glycaemic control. Advancement of a healthy oral cavity in patients with diabetes is fundamental.

\section{Funding: Nil, Conflict of interest: None Permission of IRB: Yes}

\section{References}

1. Nicki R. Colledge: Davidson's principles and practice of medicine ELBS. Vol. 17. Philadelphia: Churchill Livingstone; 1995. p. 669-774.
2. Diabetes--a global threat. Lancet. 2009 May 23;373 (9677):1735.doi:10.1016/S0140-6736(09)60954-5.

3. Daniel R, GokulanathanS, Natarajan S, Lakshmigandhan M, Kavin T. Dental Science-Short Communication; 2012;4(6);280-82.

4. 42 World Health Organization Global Prevalence of Diabetes: Estimates for the Year 2000 and Projections for 2030. Geneva:World Health Organization; 2009.

5. Saadi H, Carruthers SG, Nagelkerke N, Al-Maskari F, Afandi B, Reed R, Lukic M, Nicholls MG, Kazam E, Algawi K, Al-Kaabi J, Leduc C, Sabri S, El-Sadig M, Elkhumaidi S, Agarwal M, Benedict S. Prevalence of diabetes mellitus and its complications in a populationbased sample in Al Ain, United Arab Emirates. Diabetes Res Clin Pract. 2007 Dec;78 (3):369-77. Epub 2007 May 25.

6. Baldwin E. Oral health. Lancet. 2009 Feb 21;373(9664) :628-9. doi: 10.1016/S0140-6736(09) 60392-5.

7. Yuen HK, Wolf BJ, Bandyopadhyay D, Magruder KM, Salinas CF, London SD. Oral health knowledge and behavior among adults with diabetes. Diabetes Res Clin Pract. 2009 Dec;86 (3):239-46. doi: 10.1016 /j. diabres.2009.09.010. Epub 2009 Oct 2.

8. Al Habashneh R, Khader Y, Hammad MM, Almuradi M. Knowledge and awareness about diabetes and periodontal health among Jordanians. J Diabetes Complications. 2010;24:409-14.

9. Masood Mirza K, Khan AA, Ali MM, Chaudhry S. Oral health knowledge, attitude, and practices and sources of information for diabetic patients in Lahore, Pakistan. Diabetes Care. 2007 Dec;30(12):3046-7. Epub 2007 Aug 21.

10. Moore PA, Orchard T, Guggenheimer J, Weyant RJ. Diabetes and oral health promotion: a survey of disease prevention behaviors. J Am Dent Assoc. 2000 Sep;131 (9):1333-41.

11. Teeuw WJ, Gerdes VE, Loos BG. Effect of periodontal treatment on glycemic control of diabetic patients: a systematic review and meta-analysis. Diabetes Care. 2010 Feb;33(2):421-7. doi: 10.2337/ dc 09-1378. 
12. Lamster IB, Lalla E, Borgnakke WS, Taylor GW. The relationship between oral health and diabetes mellitus. J Am Dent Assoc. 2008 Oct;139 Suppl:19S$24 \mathrm{~S}$.

13. Saini R, Al-Maweri SA, Saini D, Ismail NM, Ismail AR. Oral mucosal lesions in non oral habit diabetic patients and association of diabetes mellitus with oral precancerous lesions. Diabetes Res Clin Pract. 2010 Sep;89(3):320-6. doi: 10.1016/j.diabres.2010.04.016. Epub 2010 May 20.

14. Preshaw PM. Periodontal disease and diabetes. J Dent. 2009 Aug;37(8):S575-7. doi: 10.1016/j.jdent. 2009.05.019. Epub 2009 May 22.

\section{How to cite this article?}

Saxena V, Saxena D, Desai A, Shrivastava S. Oral manifestations of type 2 diabetes mellitus of Bhopal city: an observational prospective study. Int J Med Res Rev 2017;5(03):293-297 doi:10.17511/ijmrr. 2017.i03.13. 